\title{
Frontières
}

\section{Restes humains et surplus de sens}

\section{Diane Laflamme}

Volume 23, numéro 1, automne 2010

Enquêtes sur le cadavre : 1. Fascination

URI : https://id.erudit.org/iderudit/1004015ar

DOI : https://doi.org/10.7202/1004015ar

Aller au sommaire du numéro

Éditeur(s)

Université du Québec à Montréal

ISSN

1916-0976 (numérique)

Découvrir la revue

Citer ce document

Laflamme, D. (2010). Restes humains et surplus de sens. Frontières, 23(1), 3-4.

https://doi.org/10.7202/1004015ar

Ce document est protégé par la loi sur le droit d'auteur. L'utilisation des services d'Érudit (y compris la reproduction) est assujettie à sa politique d'utilisation que vous pouvez consulter en ligne.

https://apropos.erudit.org/fr/usagers/politique-dutilisation/
Cet article est diffusé et préservé par Érudit.

Érudit est un consortium interuniversitaire sans but lucratif composé de l’Université de Montréal, l’Université Laval et l'Université du Québec à Montréal. Il a pour mission la promotion et la valorisation de la recherche. https://www.erudit.org/fr/ 


\section{$\begin{array}{llllllllll} & \text { E } & \text { D } & \text { I } & \text { T } & \text { O } & \text { R } & \text { I } & \text { A } & \text { L }\end{array}$}

\section{RESTES HUMAINS ET SURPLUS DE SENS}

Diane Laflamme, Ph.D., co-directrice et rédactrice en chef.

Un cadavre est fait de chair et d'os. On pourrait facilement l'oublier dans nos sociétés de haute technologie. Quand nous sommes mis en présence d'un cadavre, il s'agit le plus souvent d'un cadavre virtuel dont les pixels ne dégagent pas la moindre odeur offensante pour les narines du spectateur. La caméra ne s'y attarde pas trop pendant le bulletin de nouvelles, alors qu'au contraire elle se complaît à le montrer de très près dans les films d'horreur ou d'action ainsi que dans bien des séries télévisées diffusées aux heures de grande écoute.

Un cadavre, c'est un corps qui ne respire plus... Pourtant, notre propre capacité de respirer nous lie encore à lui, que ce respir soit un cri de surprise, un sanglot ou un soupir de soulagement. Inconnue ou familière, aimée ou haïe, c'est une personne comme moi qui est devenue... tout autre ! Et je le sais. Je me sais capable de respirer, mais incapable d'empêcher que ce souffle s'arrête un jour. Être mis en présence du cadavre, c'est faire l'épreuve de soi.

La mort me met à l'épreuve. Ce corps devenu cadavre, c'est la dépouille d'une personne qui avait sa petite histoire, qui était capable de faire des choix sensés, tout comme moi. Même s'il s'agit d'un visage inconnu entrevu sur la page d'un journal ou à l'écran, je sais que je partage avec cette personne une même destinée qui consiste à devenir un jour, moi aussi, quelque chose de tout autre. Je voudrais citer ici le choix de l'un de ces inconnus qui s'est montré capable de donner ce «tout autre » qu'à peu près tout le monde autour de moi ose à peine nommer: le cadavre! Michael Fainstat avait 87 ans lorsqu'il est décédé, à Montréal, le 29 décembre dernier. Un grand quotidien a parlé de lui dans ses pages et sur son site Web parce qu'il a fait don de son corps à la Faculté de médecine de l'Université McGill. Je ne connais rien des motifs qui ont inspiré ce choix. Ma position est celle de l'observateur. J'observe cette capacité de donner au-delà de la mort. N'y a-t-il pas là une ouverture vers le sens, et même quelque chose de «glorieux»- pour reprendre le qualificatif qu'emploie le philosophe Mikel Dufrenne quand il explique comment se manifeste le beau à partir du sensible ?

Oui : le sensible lorsqu'il est glorieux, n'est jamais insigni-

fiant; ce qui se dit par lui n'est pas un concept ou une idée,

c'est un monde. Un monde, c'est-à-dire un signifié sans

contours, un horizon de sens, plutôt qu'un sens déterminé, mais que pourtant le sentiment identifie et reconnaît sans

peine. (1995, p. 32)

Les restes humains peuvent «signifier» parce qu'ils peuvent nous ouvrir un monde à même le sensible. C'est justement ce qui caractérise les phénomènes qui font sens: ils donnent accès à plus vaste que ce qui se montre. 
Observant ce que nous appelons notre expérience, le sociologue Niklas Luhmann constate qu'elle fait toujours référence à quelque chose d'autre, en plus de s'actualiser comme "cette» opération consciente ou «cette» opération de communication, plutôt qu'une autre. Luhmann attribue la capacité de constituer le sens à deux types de systèmes: les systèmes psychiques (conscients) et les systèmes sociaux. Un système psychique est capable de penser. Un système social est capable de communiquer. Ce que Luhmann appelle «le sens», c'est la solution que propose le système psychique ou le système social alors en cause pour s'accommoder de la coexistence de ces deux aspects dans l'expérience humaine : son contenu actuel, qui actualise l'un de mes choix, et tout ce à quoi cette expérience fait référence en plus de son contenu actuel, c'est-à-dire le monde qui se donne comme horizon de sens (Luhmann, 1990a, p. 25 ; 1990b).

Luhmann constate qu'en tant que "phénomène", le sens se constitue comme en excès par rapport à lui-même: "Quelque chose se tient là, au point focal, au centre de l'intention, et tout le reste est indiqué de façon marginale, comme l'horizon d'un "et ainsi de suite" indissociable de l'expérience et de l'action» (1995, p. 60 ; notre traduction). Il y a un «surplus » de références à d'autres possibilités d'expérience et d'action. Le sens renvoie à ce qui est choisi ici et maintenant, parmi toutes les possibilités, tout en préservant mon accès à un monde qui ne rétrécit pas à mesure que j'y fais des choix (Luhmann, 1995, p. 61).

Le sens est enveloppé en lui-même, poursuit le philosophe Jean-Michel Salanskis. Cet enveloppement du sens serait l'une des caractéristiques de «l'effet-de-sens » que le développement de la pensée herméneutique a permis de dégager:

L'enveloppement du sens est sa structure décisive. Ce qui fait que le sens porte l'existence, la temporalise, qu'il passe et qu'il oriente, c'est qu'il est enveloppé en luimême, que chaque occurrence de l'adresse porte plus de sens qu'elle n'en signifie. Même si aucune élucidation de l'excès du sens dans son occurrence n'a lieu, cet excès est constitutif du sens comme tel. Une occurrence de sens absolument transparente ne serait pas occurrence de sens, mais exhibition d'objet, présentation pleine. (1997, p. 417-418)

L'exhibition de cet objet qu'est un cadavre met l'observateur à l'épreuve. Mis en présence de restes humains, où faut-il chercher le surplus de sens? Si le sens oriente, vers quel lieu me dirige-t-il ?

Luhmann et Salanskis s'inscrivent tous deux dans le courant de la phénoménologie husserlienne et de ses propositions sur la constitution $\mathrm{du}$ sens. La constitution du sens à partir de nos pensées conscientes et de nos communications n'est pas un banal processus, parmi tant d'autres. Ce qui est en jeu, c'est qui nous sommes, qui nous devenons quand nous exerçons notre capacité de penser et de communiquer les uns avec les autres, constate Luhmann. Le sens oriente parce qu'il est enveloppé de toutes parts plutôt que transparent, ajoute Salanskis. Penser et communiquer permettent à notre conscience de se déployer dans un univers de sens. Pourtant, notre conscience n'a pas accès aux processus biologiques qui lui permettent d'exister; ils se déploient autour d'elle, mais sans elle.

Ce qui enveloppe mon souffle est fait de chair et d'os qui sont miens. Comme le sens, nous sommes enveloppés dans ce qui nous fait et en même temps nous dépasse. S'il y a une inscription corporelle du sens, nous en sommes le lieu.

\section{Bibliographie}

DUFRENNE, M. (1995). «Art. A. Le beau », Encyclopcedia universalis, Paris, Encyclopædia universalis, Corpus 3, p. 29-35.

JOHNSTON, D. (2011). «Fainstat's last gift was himself. Former city councilor donates his body to McGill's medical school so students can learn about human anatomy ", The Gazette, 8 janvier, p. A3.

LUHMANN, Niklas (1995). Social Systems, Stanford, Stanford University Press.

LUHMANN, Niklas (1990a). Essays on Self-Reference, New York, Columbia University Press.

LUHMANN, Niklas (1990b). «Développements récents en théorie des systèmes", dans G. Duprat, Connaissance du politique, Paris, PUF.

SALANSKIS, Jean-Michel (1997). « Herméneutique et philosophie du sens ", dans J.-M. Salanskis, F. Rastier et R. Scheps, Herméneutique : textes, sciences, Paris, PUF.

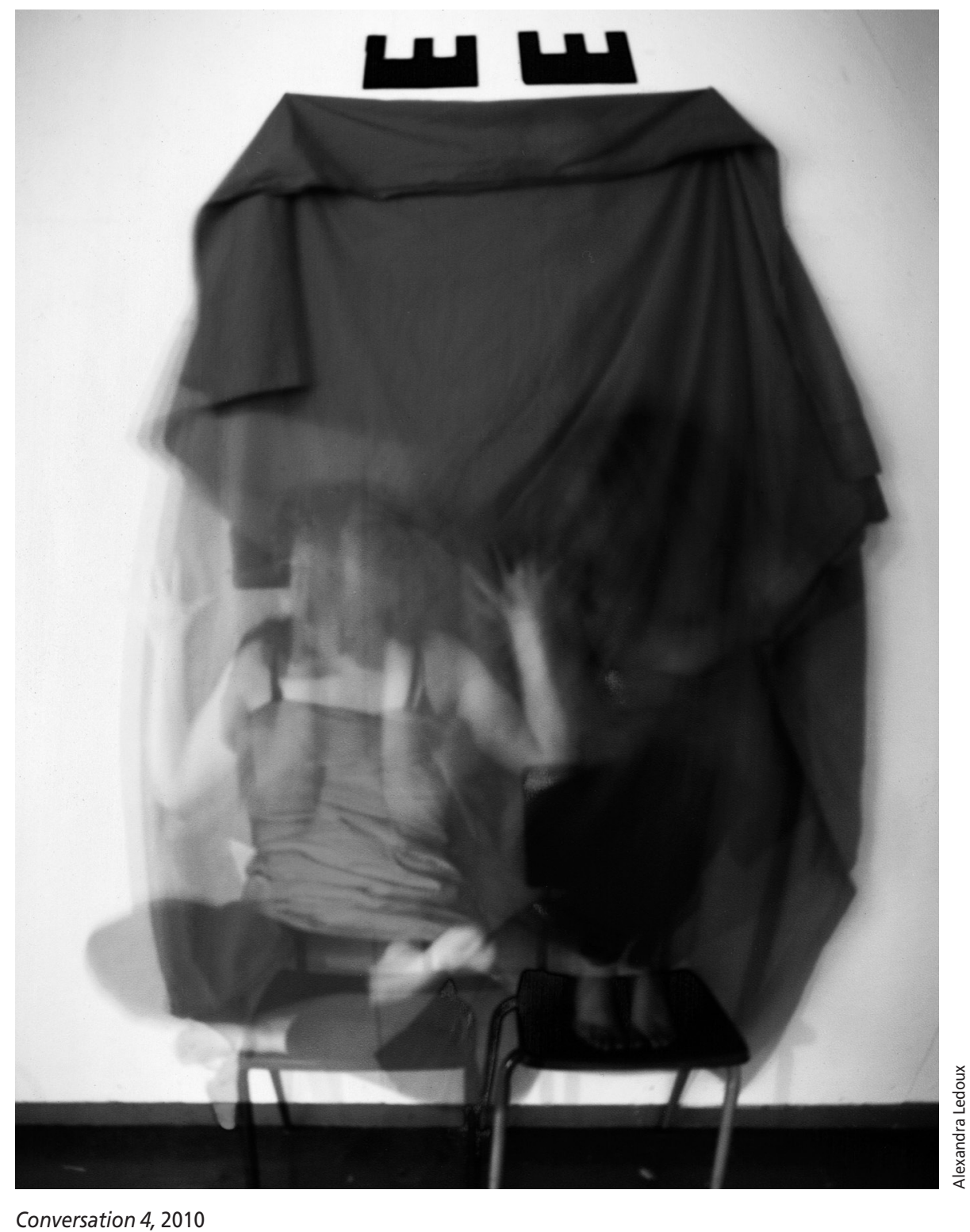

